\title{
Design of a Flexible Hardware Interface for Multiple Remote Electronic Practical Experiments of Virtual Laboratory
}

http://dx.doi.org/10.3991/ijoe.v8iS2.2004

\author{
S. Farah ${ }^{1}$, A. Benachenhou ${ }^{1}$, G. Neveux ${ }^{2}$ and D. Barataud ${ }^{2}$ \\ ${ }^{1}$ LEOG, University of Mostaganem, Mostaganem, Algeria \\ ${ }^{2}$ XLIM, University of Limoges, Limoges, France
}

\begin{abstract}
The objective of this work is to present a new design of a Flexible Hardware Interface (FHI) based on PID control techniques to use in a virtual laboratory. This flexible hardware interface allows the easy implementation of different and multiple remote electronic practical experiments for undergraduate engineering classes. This interface can be viewed as opened hardware architecture to easily develop simple or complex remote experiments in the electronic domain. The philosophy of the use of this interface can also be expanded to many other domains as optic experiments for instance. It is also demonstrated that software can be developed to enable remote measurements of electronic circuits or systems using only Web site Interface. Using standard browsers (such as Internet explorer, Firefox, Chrome or Safari), different students can have a remote access to different practical experiments at a time.
\end{abstract}

Index Terms-Ajax, flexible hardware interface, practical experiments, practical evaluation board, Remote Virtual Lab, VLAN, web interface.

\section{INTRODUCTION}

Many online remote laboratories [1] have been developed since the beginning of 90ties. Most of them are specialized in hardware architecture [2]-[7], in software [8]-[11]. Some of them have developed specific techniques to facilitate the access and the management of resources [11]-[15]. Most of the virtual laboratories are specialized for a specific domain or field. The expansion of these virtual laboratories to other technical domain or field is not so easy to elaborate [2].

A virtual Microwave and Optical laboratory (LABoratoire d'ENseignement VIrtuel : LAB-EN-VI) has been developed at Limoges University, based on an accurate undergraduate teaching laboratory concerning optic and microwave fields. This LAB-EN-VI addresses several learning issues as simulation and experiment. To give a remote access control of different practical experiments in microwave and optical fields is of prime importance regarding the high costs of equipments (Sources, analyzers, receivers,...) involved in experimental labs. University education cannot provide one of these costly equipment's by students. So, one solution is the ability for multiple students to share a single instrument or test bench: a remote virtual lab can afford this solution.
This paper describes the design of a Flexible Hardware Interface (FHI) based on PID control techniques and Web service for making remote electronic practical experiments for several users at a time through the World Wide Web (WWW). Connected to this hardware interface, different "practical evaluation boards" (PEB) have been designed and developed. Each PEB is an electronic circuit illustrating a theoretical principle. The philosophy of this open architecture enables everyone to design a PEB which can be connected to the FHI. For each FHI, a specific web page can be then easily elaborate to help students and their teacher to realize the associated practical experiment. Remote students with standard browsers monitor their results for each PEB in real time from their computer. The FHI permits to select the PEB from the web service page.

Section II of this paper describes the virtual microwave laboratory LAB-EN-VI and its network and software configurations. Section III presents the full design of the FHI and the PEB associated to specific applications of remote electronic practical experiments. Section IV gives examples of web interfaces pages.

\section{DESCRIPTION OF THE VIRTUAL LAB "LAB-EN-VI"}

The virtual Microwave and Optical laboratory (LABoratoire d'ENseignement VIrtuel : LAB-EN-VI) is now created, developed and used by students in the undergraduate engineering courses of the master ARTICC (Architecture des Réseaux et Technologies Induites des Circuits de Communication : Network Architectures and Associated Technologies of Communication Circuits) at the universities of Limoges and Mostaganem. The courses focus on microwave and optical sub-systems and circuits used in different telecommunication systems (GPS, UMTS, DVB, Radar applications ...). The network architecture of this LAB-EN-VI is described in Fig. 1. It is based on different local area networks (LAN) provided by the IT departments of the University of Limoges and the XLIM laboratory. Remote students with a unique login and password provided by the CAS server can access the remote theoretical courses available on the Moodle server. With this LAB-EN-VI, they can also use several professional software, available on different application servers (ADS, Labview, Matlab, CST, HFSS...) and managed by a license server hosted XLIM 


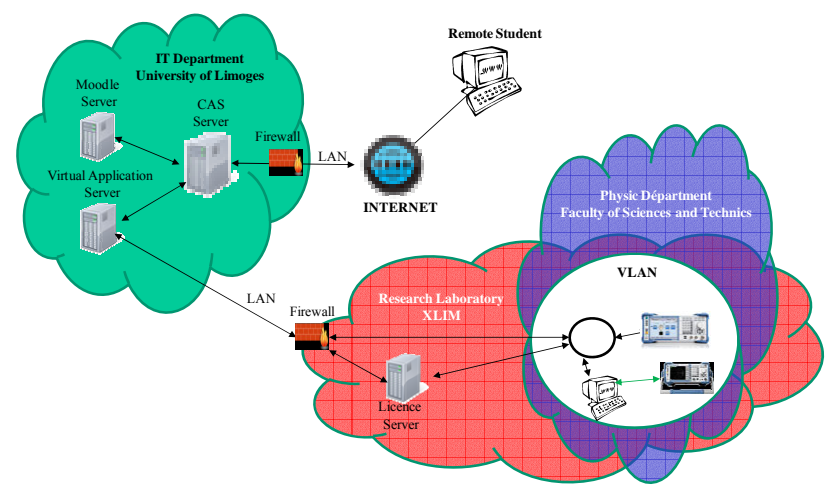

Figure 1. Architecture of the microwave remote virtual Lab

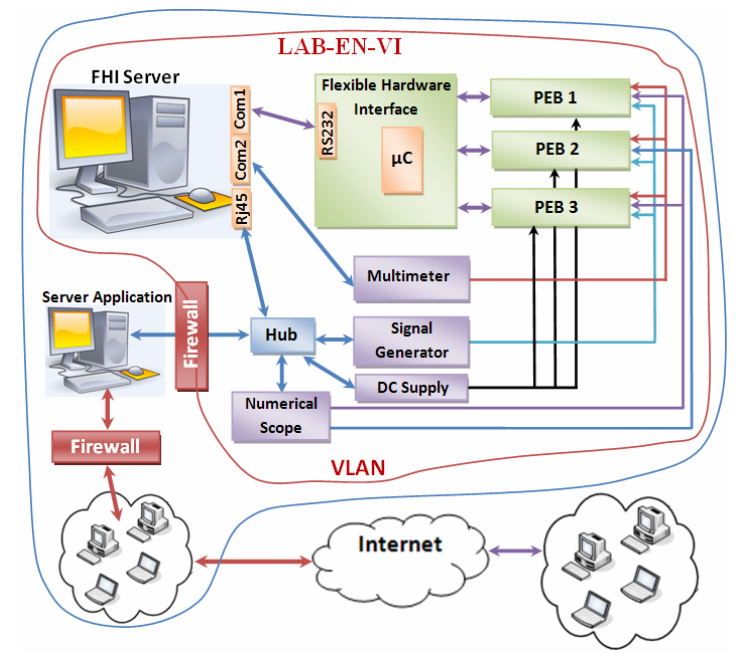

Figure 2. Description of the FHI and PEB in the microwave remote virtual Lab

To complete understanding of their theoretical course material, the LAB-EN-VI provides them different lab experience by controlling remote electronic practical experiments. In such context, it is highly necessary to provide them the capability to work on different experiments based on an identical architecture. This is why a common interface "Flexible Hardware Interface" has been developed capable of driving several "Practical Evaluation Boards" (PEB), as shown in Fig. 2. This architecture facilitates the study, design and implementation of practical experimental courses.

\section{A. Hardware and Network configuration}

With the homemade Flexible Hardware Interfaces (FHI) and practical evaluation boards (PEB), it is very easy to configure and control remotely the same instruments used for several experiments. It is now possible to use only one Vector Network Analyzer, one Digital Sampling Scope or only one microwave generator for different practical experiments thanks to this FHI as demonstrated in Fig. 2. This architecture allows working with multiple experiments without a prohibitive cost associated with the proliferation of instruments for experimentation.

Another advantage of this FHI leads in the fact that it is independent of the connectivity interfaces of instruments: it permits to talk with either serial interface (RS 232, USB...), either GPIB or RJ45, as described in Fig. 2.

More and more instruments have their own embedded web server with RJ45 interface, giving remote users the

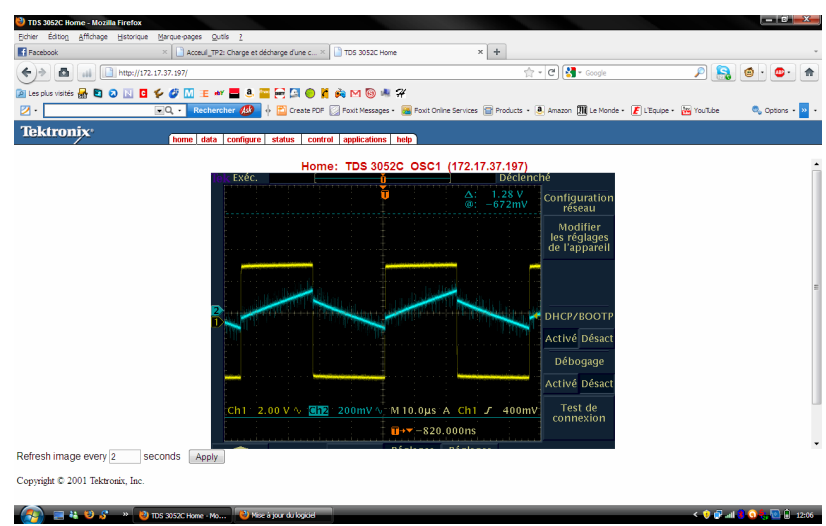

Figure 3. Example of web interface for instrument with embedded web server (RJ45 interface)

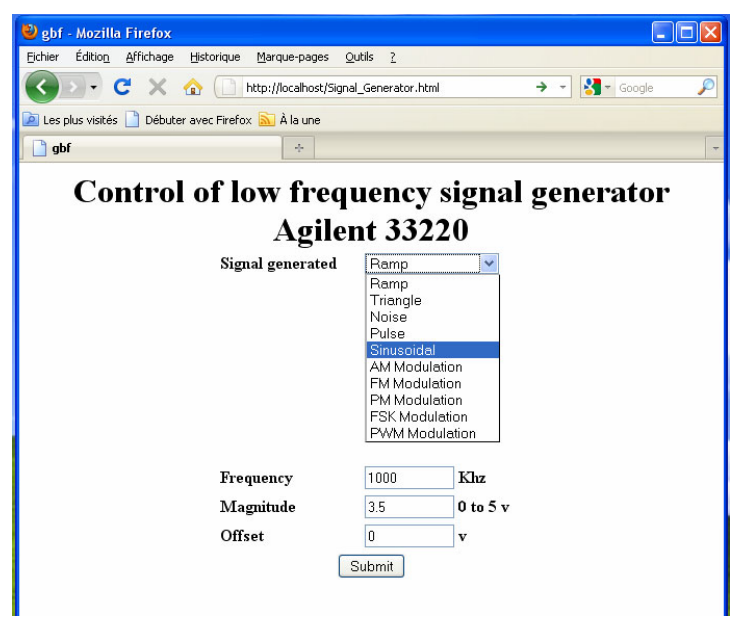

Figure 4. Example of web interface for PEB

capability to use a remote display for controlling these instruments as described in Fig. 3

The main drawback to this remote working mode is that it is difficult to incorporate this remote display in a more complex practical experiment using different instruments and theoretical concepts. The FHI, connected to a specific server, is able to integrate different instruments in the same unique web page to give to remote students the capability to work on specific practical experiments within the use of the PEBs as shown in Fig. 4. It represents then a lightweight web service for the full practical experiment.

Each PEB requires then the design of a web interface based on the Ajax approach [16] to transfer the main commands from the web pages to the PEBs through the FHI. A PHP [17] service is installed in the FHI server. With this PHP service graphical objects can be inserted in web pages representing the response obtained from of practical evaluation boards (PEB).

\section{B. Software configuration}

The associated software architecture is based on a new web interface design using HTML (CSS) [18] and JavaScript language [19]. With these web interfaces, easy handling of remote practical evaluation boards (PEB) is provided. The PEB is graphically represented in the web pages. Each PEB contains different configurations. The choice of one configuration is provided to remote student through the web page as represented in Fig. 5. Remote students handle radio buttons in the web interface to 
configure the PEB. The image of the configuration is then modified by the student and the result is directly applied in real time to the PEB. Each configuration is binary coded. The FHI receives the code from the web page to configure correctly the PEB. The web page also allows setting the signal sources and enables the configuration of the receivers (Digital sampling scope for instance).

To ensure flexibility in the transfer of commands from remote student computer to $\mathrm{PEB}$, the Ajax widely used technique has been adapted. Employing Ajax approach avoids the problem of execution of PHP full pages which can complicate the design of the web interfaces as described in Figure 6.

It also avoids delays in the transfer protocol to the FHI and then to practical evaluation boards (PEB). The PHP server connected to the FHI consider the serial Port (RS232) as a simple file so that all PHP file functions can be applied to the serial interface. The different codes associated to the PEB configurations are sent to the FHI through this Serial Port File.

\section{DESCRIPTION OF THE FLEXIBLE HARDWARE INTERFACE AND PRACTICAL EVALUATION BOARDS}

\section{A. Flexible Hardware Interface (FHI)}

The hardware part of the architecture is based on the realization of the Flexible Hardware Interface (FHI) which controls the practical evaluation boards (PEB). The FHI is primarily designed using a the microcontroller to communicate with the FHI server via the RS232 interface. Te microcontroller also ensures the configuration of the different PEB by controlling different relay as represented in Fig. 7.

The Fig. 8 shows photographs of the built FHI and the result of the PROTEUS simulation software.

Fig. 9 shows a photograph of the FHI and a specific PEB which is dedicated to a theoretical illustration of charge and discharge of capacitance.

\section{B. Practical Evaluation Board (PEB)}

The PEB represents the second part of the architecture. It is specific electronic boards developed to illustrate a theoretical electronic principle in this case. For instance, a specific PEB can be created to show the influence of resistances in the measurement and the theoretical comparison of rise time and fall time in a charge and discharge process capacitance. The practical board is simply designed with 2 resistances and 2 capacitances and connectors as represented in Fig. 10. Following the web configuration realized by the remote student, the FHI will switch the different relay to connect the chosen resistance and capacitance. The remote student can then observe the different curves on the connected sampling scope.

The PEBs are designed in order to let remote student trying all possible circuit configurations as if he's in the real lab as represented in Fig. 11. All configurations are managed by relays controlled by the microcontroller of the FHI receiving corresponding commands from the FHI server.

Fig. 12 represents different PEBs already realized to control and to characterize different Diodes Fig.12 (1), to measure rise time and fall time in charge/discharge of capacitance process Fig.12 (2), to understand working modes of operational amplifiers Fig.12 (3).

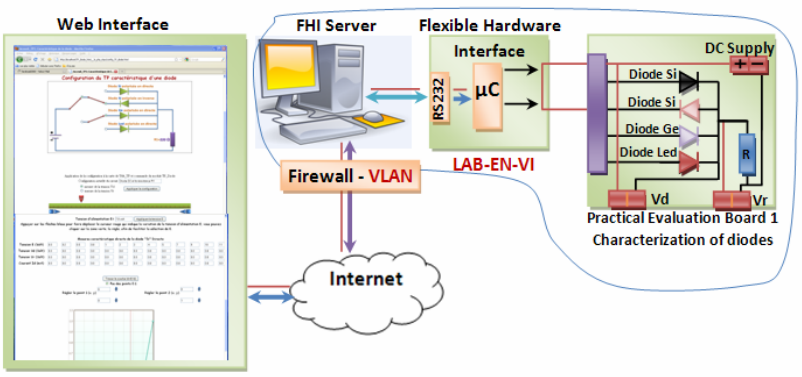

Figure 5. Example of web interface configuration of the PEB.

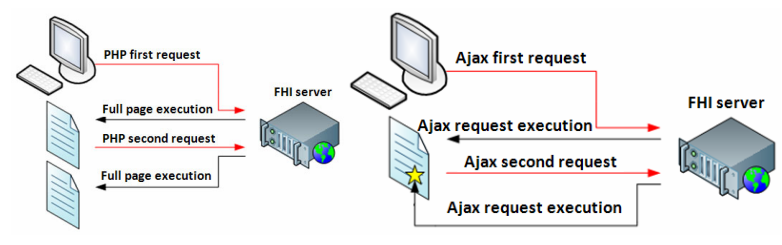

Figure 6. Web site using:

1. PHP interface

2. HTML pages and AJAX approach

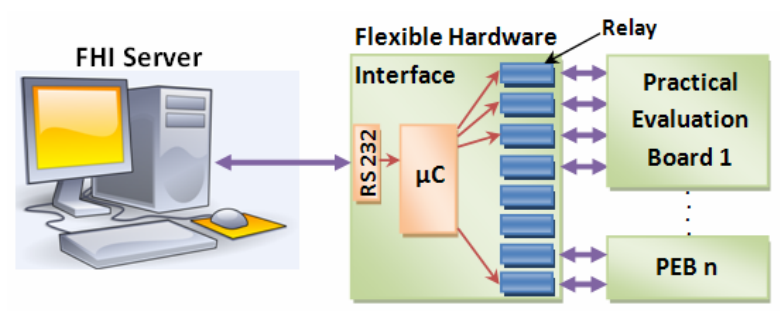

Figure 7. Relay configuration of the Flexible Hardware Interface.

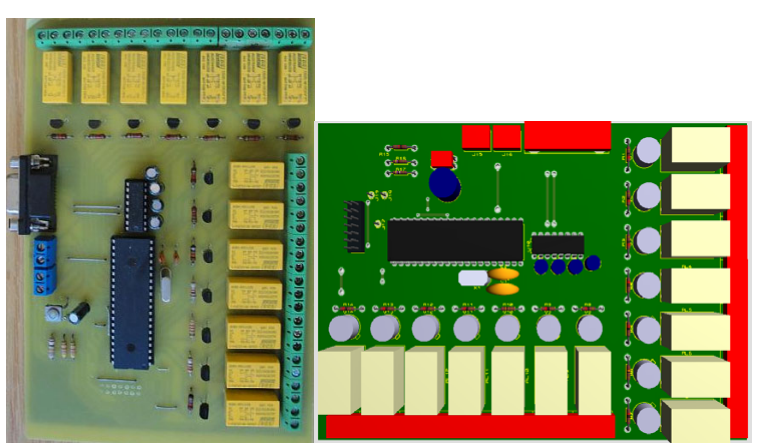

Figure 8. Flexible Hardware Interface, real and 3D simulation view

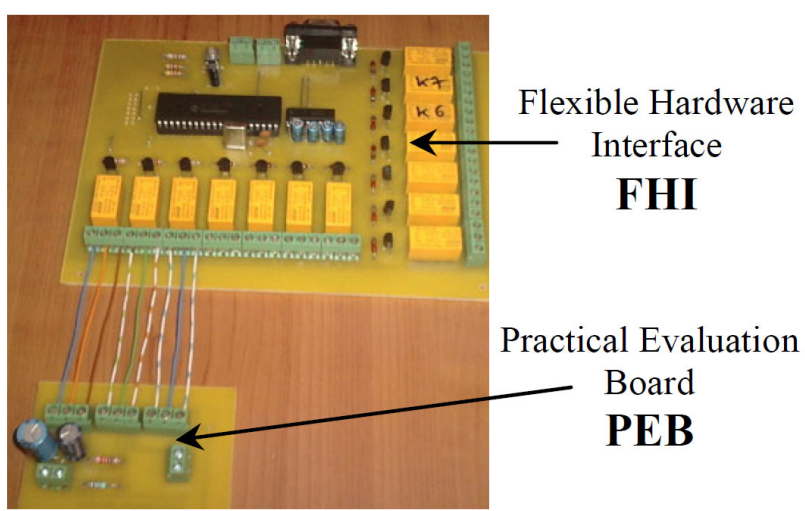

Figure 9. Connection between the Flexible Hardware Interface and practical evaluation board 


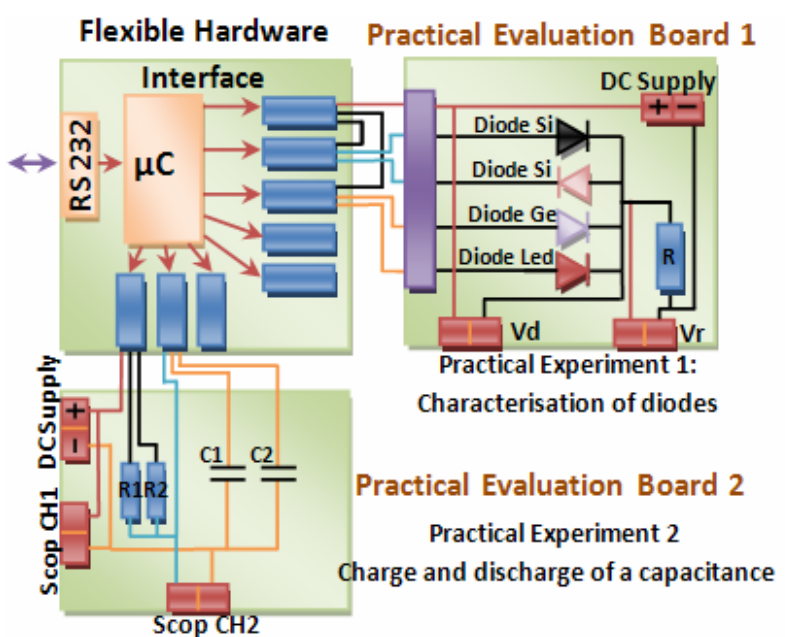

Figure 10. Operating diagram of practical evaluation boards

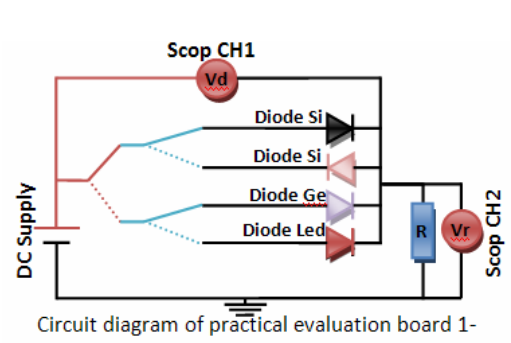

Characterization of diodes

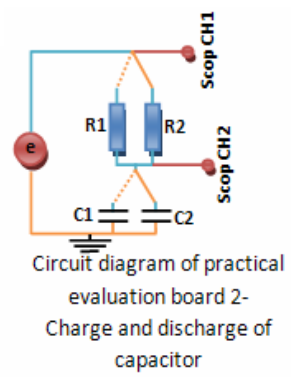

Figure 11. Some Electrical schematics of practical evaluation boards
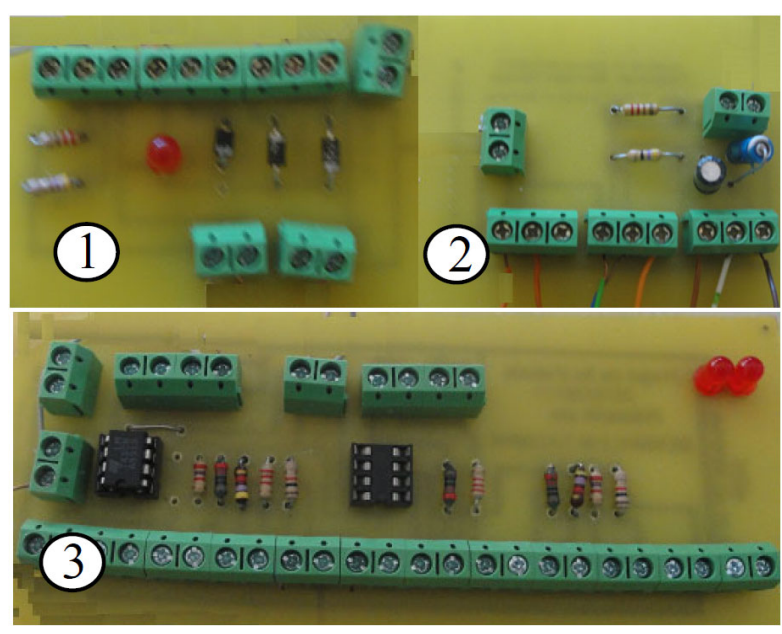

Figure 12. Examples of realized practical evaluation boards

1. Characterization of diodes

2. Charge and discharge of capacitor

3. Operational amplifier

Fig. 13 represents the full test bench experiments in the LAB-EN-VI with the FHI, a PEB and all required instruments (DC supply, signal generator and sampling scope in that case).

\section{Communication protocol}

The proposed protocol ensures the communication between the web interface and the FHI. It can manage multiple users using multiple PEBs. Fig. 14 shows how the software successfully manages two remote students work-

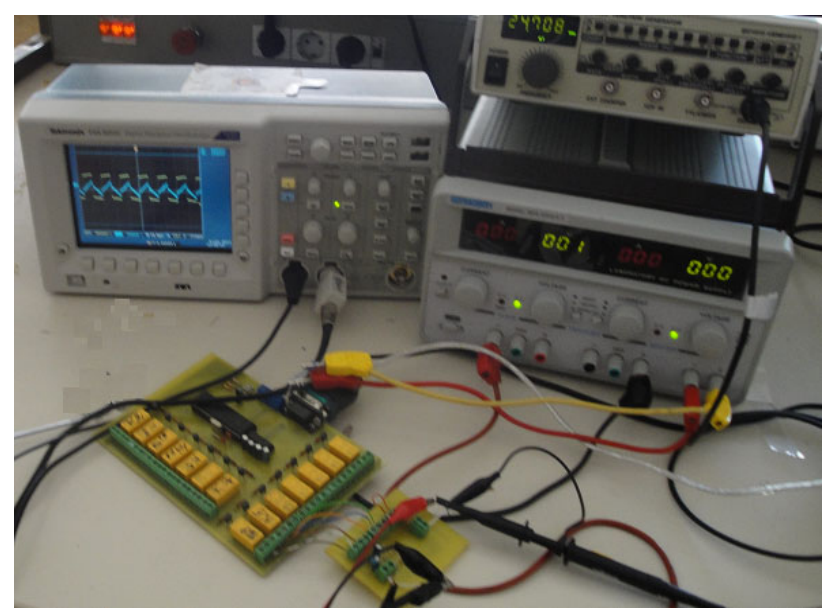

Figure 13. Example of full test-bench

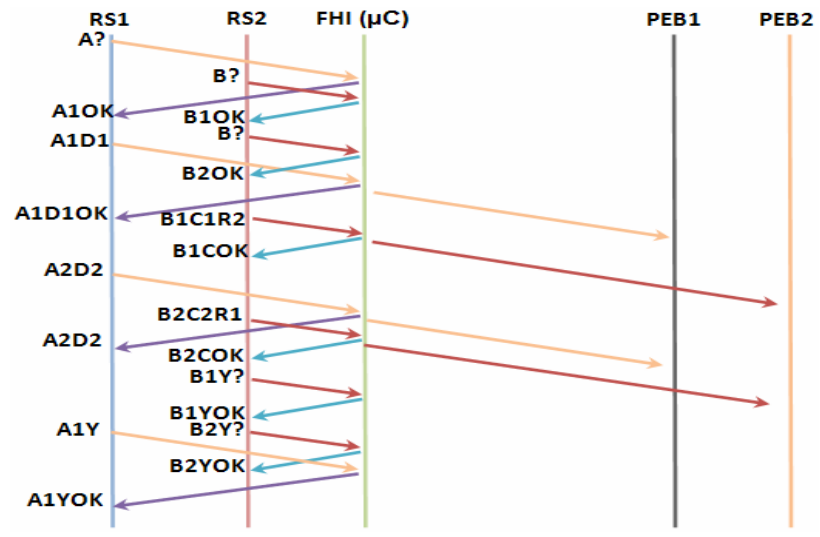

Figure 14. Description of the communication protocol between web interface Flexible Hardware Interface and 2 PEB for 2 remote students (RS).

ing with two different PEB connected to the same FHI. This protocol is simply expandable to several studentsworking at the time with several PEB connected to a unique FHI. In the case of two remote students (RS1 and RS2) and two PEB (PEB1 and PEB2), it can be viewed that the different requests are sequentially treated to conduct the tests in order.

\section{EXAMPLE APPLICATION OF THE FHI IN THE LAB-EN-VI}

\section{A. Design of the web interface}

The web interface designed for the remote virtual lab is based on one or more configurations of PEBs as described previously. Web pages are written in HTML "CSS - style" and JavaScript languages. JavaScript is used to facilitate interaction with the user and to ensure communication with Ajax.

To simplify learning, visual objects are defined in the web pages such as buttons, radio buttons and images. With this graphical approach (provided by style pages "CSS" for formatting web pages), it is easy for students to understand and to simulate the control of the remote relays.

Finally, web pages are not voluminous leading to a lightweight web service, which facilitates the loading of their objects. It also can work with slow Internet connections. The control and configuration of the instruments with embedded server is included in the web pages as 
specific links. To draw the different curves obtained from measurements, graphs function are done using the free graphics library "jQuery-Flot"[20] written in JavaScript.

The educational content of practical "Introduction, purpose and results" remains as the one in reel laboratory as represented in Fig. 15.

\section{B. Examples of web interfaces}

Two examples of web interfaces are now presented with their images showing the remote laboratory tests.

The Fig. 16 (1) shows the layout design of a practical experiment to study the DC characteristics of diodes. First, Remote student choose the diode they would like to characterize among the four diodes. They modify the value of the power supply. They choose the voltage they want to measure (Resistance voltage or diode voltage). For each value of DC supply they complete the array in the web page. Once the array completed they can draw the curve on the same web page. They can verify the results by comparing with the following theoretical equation:

$$
I_{d}=I_{s}\left(1-e^{\frac{q V_{d}}{k T}}\right)
$$

Is : saturation current

$\mathrm{q}=1,6.10-19 C$, electron charge

$\mathrm{k}=1,38.10-23 \mathrm{~J} / \mathrm{K}$, Boltzmann constant

$\mathrm{T}$ : Temperature (Kelvin)

Vd : Diode voltage

The Fig. 16 (2) shows the practical experiment to study the charge and discharge of capacitance; it uses the same procedure as before to select resistance and capacitance. Remote student have also to configure the signal generator and the oscilloscope to perform the measurements.

If $\mathrm{C}$ is the capacitance and $\mathrm{R}$ the resistance, the differential equations linking the current to the difference potential Uc ( $t$ ) across the capacitance are:

- Solution of the discharge:

$$
U_{c}(t)=E e^{-\frac{t}{R C}}
$$

- Solution of the charge:

$$
U_{c}(t)=E\left(1-e^{-\frac{t}{R C}}\right)
$$

(3)

The web interface can easily be integrated in a remote learning platform as the "Moodle" platform used for ARTICC by inserting a link to the interface as described in Fig. 17. The access to the instruments via a specific VLAN has been explained in section II.

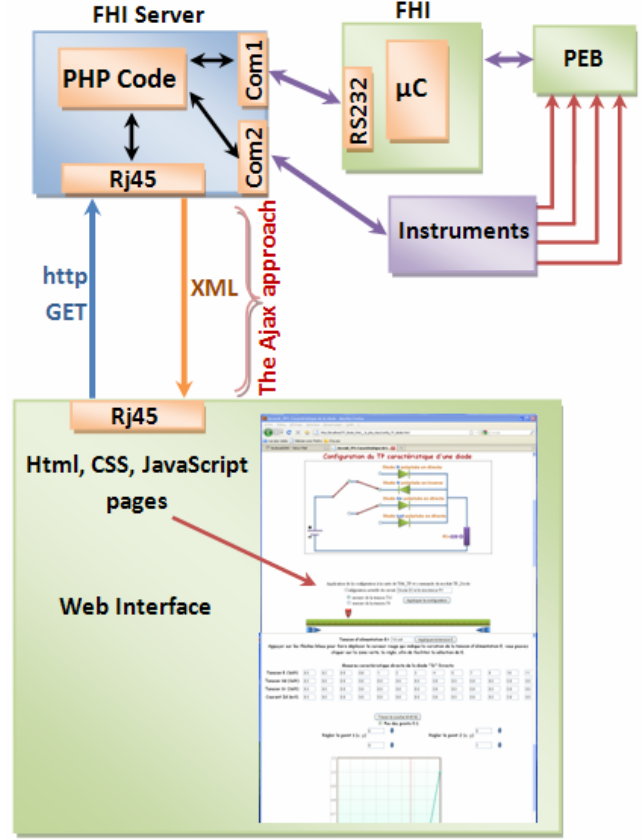

Figure 15. Example of web Interface communicating with the FHI server, the FHI, the instruments and the PEB.

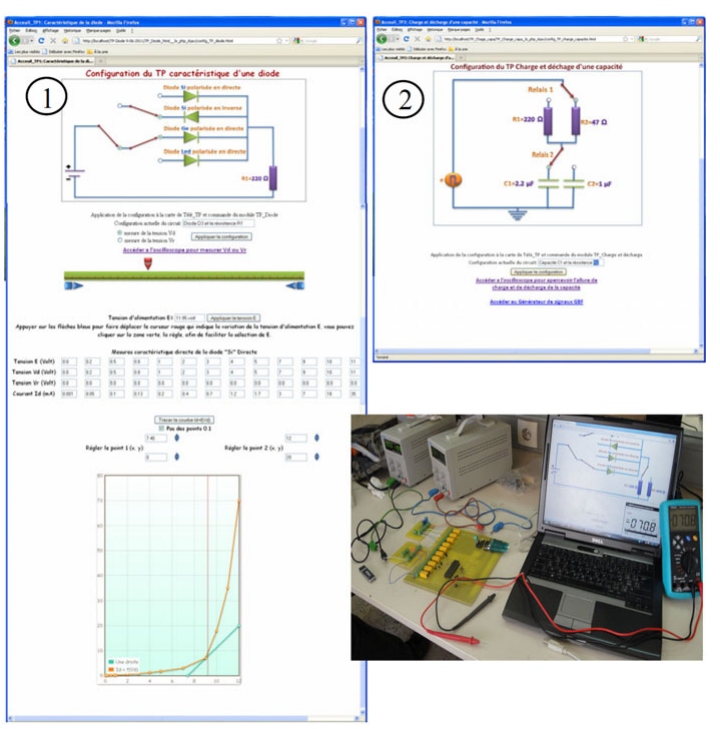

Figure 16. Overview and test of web interfaces

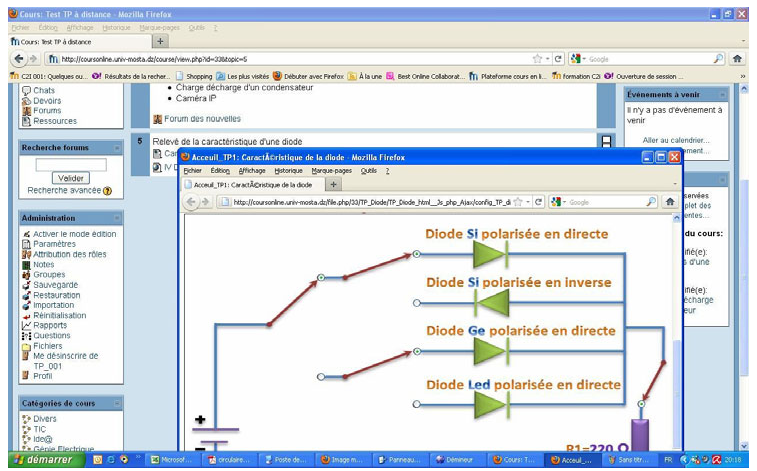

Figure 17. Integration of remote practical experiments in Moodle platform. 


\section{CONCLUSION}

In this paper, the design of a Flexible Hardware Interface (FHI) associated with different "practical evaluation boards" (PEB) has been presented. Linked to specific web service, the FHI and PEB allow undergraduate engineering students performing remote electronic practical experiments. It has been demonstrated that the main advantage of this technology is the fact that it is a fully open architecture. Effectively, it enables everyone to design a PEB which can be connected to the FHI. This concept facilitates the integration of new practical experiments for several fields. It has also be shown that multiple users can access to the same practical evaluation boards (PEB) by using a simple web interface HTML type. Flexible Hardware Interface (FHI) and its microcontroller are reprogrammable directly from the FHI server which facilitates modifications and adaptations of new architecture practical experiments.

The proposed web pages are based on HTML and JavaScript languages, which facilitate future integration to other practical evaluation boards (PEB). These interfaces are lightweight and flexible, so adaptable to slow Internet connections. The practical experiments are "multi-user" and remote students can access to them in an educational Moodle server.

The integration of others disciplines to our practical remote lab's is to be the subject of our future work.

\section{REFERENCES}

[1] P. Bauer, V. Fedak, V. Hajek "Distance and Virtual Laboratories" Joint Czech- Polish - Slovak Conference under the framework of project GACR N ${ }^{\circ}$ 102/06/1320 "Optimization of Small Electrical Machine", 14-16 July 2008.

[2] J. Santos, J. Mendonça and J. C. Martins, "Instrumentation Remote Control through Internet with PHP”, VECIMS 2008 IEEE International Conference on Virtual Environments, HumanComputer Interfaces, and Measurement Systems Istambul, Turkey, 14-16 July 2008.

[3] R. Sallier, R. Mißler, B. Brück and A. Schütze, "VenDASys - A Versatile Experimentation Platform for Educational Purposes", International Journal of Online Engineering (iJOE), iJOE - Volume 6, Issue 3, August 2010

[4] J. García-Zubia, I. Angulo, J. Irurzun, P. Orduña, J. Ruiz, U. Hernández, M. Castro and E. Sancristobal, "Easily Integrable Platform for the Deployment of a Remote Laboratory for Microcontrollers”, International Journal of Online Engineering (iJOE), iJOE - Volume 6, Issue 3, August 2010.

[5] E. Lunca, S. Ursache and O. Neacsu, "Graphical Programming Tools for Electrical Engineering Higher Education", International Journal of Online Engineering (iJOE), iJOE - Volume 7, Issue 1, February 2011.

[6] E. Lunca, S. Ursache and O. Neacsu, "Graphical Programming Tools for Electrical Engineering Higher Education", International Journal of Online Engineering (iJOE), iJOE - Volume 7, Issue 1, February 2011.

[7] Ch. Tsihouridis, D. Vavougios and G. S. Ioannidis, S. Paraskeuopoulos1, "Specially Designed Sound-Boxes Used by Students to Perform School-Lab Sensor-Based Experiments, to Understand Sound Phenomena", International Journal of Online Engineering (iJOE), iJOE - Volume 7, Issue 1, February 2011.
[8] P. Bisták and P. Folvarčík, "Remote Laboratory Java Server Based on JACOB Project", International Journal of Online Engineering (iJOE), iJOE - Volume 7, Issue 1, February 2011.

[9] E. Lunca, S. Ursache and O. Neacsu, "Adaptive Follow-Up of Online Engineering Laboratories Activities", International Journal of Online Engineering (iJOE), iJOE - Volume 7, Issue 1, February 2011.

[10] C. Gravier and N. Abdellaoui, "Adaptive Follow-Up of Online Engineering Laboratories Activities", International Journal of Online Engineering (iJOE), iJOE - Volume 6, Issue 3, August 2010.

[11] C. Riman, A. El Hajj and I. Mougharbel, "A Remote Lab Experiments Improved Model", International Journal of Online Engineering (iJOE), iJOE - Volume 6, Issue 2, May 2010.

[12] S. P. Satsangee, R. Mohd and R. Gandhi, "Remote Electroanalytical Laboratory", International Journal of Online Engineering (iJOE), iJOE - Volume 7, Issue 1, February 2011.

[13] A. Abu-aisheh and T. Eppes, "Remote and Virtual Instrumentation Platform for Distance Learning", International Journal of Online Engineering (iJOE), iJOE - Volume 6, Issue 3, August 2010.

[14] K. DeLong, V. J. Harward, P. Bailey, J. Hardison, G. Kohse and Y. Ostrocsky, "Three Online Neutron Beam Experiments Based on the iLab Shared Architecture", International Journal of Online Engineering (iJOE), iJOE - Volume 7, Issue 1, February 2011.

[15] S. Dutta, S. Prakash, D. Estrada and E. Pop, "A Web Service and Interface for Remote Electronic Device Characterization”, IEEE TRANSACTIONS ON EDUCATION.

[16] http://www.adaptivepath.com/ideas/ajax-new-approach-webapplications "Ajax: A New Approach to Web Applications", February $18,2005$.

[17] http://www.php.net/manual/fr/intro-whatis.php "Qu'est ce que PHP? ”, Manuel PHP, Mehdi Achour, Friedhelm Betz , Antony Dovgal, Nuno Lopes, Hannes Magnusson, Georg Richter, Damien Seguy, Jakub Vrana, 2011-06-17.

[18] http://tools.ietf.org/html//rfc1866 "hypertext markup language 2.0", t. berners-lee, d. connolly, november 1995.

[19] http://fr.wikipedia.org/wiki/Javascript "JavaScript - The Definitive Guide", Fourth Edition, David Flanagan, éditions O'Reilly Media, Sebastopol, Californie.

[20] http://www.kicoe.net/blog/web-internet/flot-une-librairie-degraphes-pour-jquery-1646 "Flot - Une librairie de graphes pour JQuery".

\section{AUTHORS}

S. F. Author is with the Electrical Engineering Department, University of Mostaganem, Bp227- 27000 Mostaganem, Algeria (e-mail: said.farah@etu.unilim.fr).

A. B. Author is with the Electrical Engineering Department, University of Mostaganem, Bp227- 27000 Mostaganem, Algeria (e-mail: abdelhalim.benachenhou.aff@unilim.fr).

G. N. Author is with XLIM laboratory, University of Limoges, 123 Av. A. THOMAS 87050 LIMOGES, France (e-mail: guillaume.neveux@xlim.fr).

D. B. Author is with XLIM laboratory, University of Limoges, 123 Av. A. THOMAS 87050 LIMOGES, France (e-mail: denis.barataud@xlim.fr).

This work was supported by Calouste Gulbenkian Foundation, Lisbon, Portugal. It is an extended version of a presentation given during the 1st Experiment@ International Conference, 17/18 November 2011 in Lisbon, Portugal. Manuscript received 29 February 2012. Published as resubmitted by the authors 18 March 2012.. 